\title{
Electron-beam collimation with a quantum point contact
} \author{
Philips Research Laboratories, 5600 JA Eindhoven, The Netherlands \\ C. J. P. M. Harmans \\ Delft University of Technology, 2600 GA Delft, The Netherlands \\ C. T. Foxon \\ Philips Research Laboratories, Redhill, Surrey, RH1 5HA, England
}

L. W. Molenkamp, A. A. M. Staring, ${ }^{*}$ C. W. J. Beenakker, R. Eppenga, C. E. Timmering, and J. G. Williamson

(Received 12 July 1989)

\begin{abstract}
Collimation of the electron beam injected by a point contact in a two-dimensional electron gas is demonstrated using a geometry with two opposite point contacts as injector and collector. The collimation is maintained over a distance of at least $4 \mu \mathrm{m}$, and is destroyed by a small magnetic field. The inferred collimation factor scales linearly with the point-contact resistance, as predicted by the semiclassical theory.
\end{abstract}

Recently, Wharam et al. ${ }^{1}$ reported on the nonadditivity of the series resistance of two opposite quantum point contacts in a two-dimensional electron gas (2D EG). This phenomenon was later discussed by Beenakker and van Houten $^{2}$ in terms of collimation of the electron beam injected by a point contact. In addition, Baranger and Stone $^{3}$ argued that such collimation effects are responsible for the quenching of the Hall resistance in very narrow channels. ${ }^{4}$ Experimental support for this explanation was given by Chang, Chang, and Baranger. ${ }^{5}$

Neither a series resistance nor a Hall resistance measurement gives direct information on the degree of collimation. In view of the importance of collimation for transport in small structures, we have decided to study this effect directly, using two opposite point contacts as injector and collector of an electron beam with an adjustable degree of collimation. We will show that these collimation effects can be well understood using a semiclassical simulation of the transport through the device.

For sample fabrication, we employ electron-beam lithography in a polymethylmethacrylate double-layer resist (using a Philips EBPG-4 Beamwriter) and lift-off techniques to deposit gold gates on top of a previously fabricated Hall-bar structure. We have fabricated two different types of microdevices on a GaAs/(Al,Ga)As heterojunction wafer with a 2D EG mobility of about 100 $\mathrm{m}^{2} \mathrm{~V}^{-1} \mathrm{~s}^{-1}$. Both devices consist of a narrow channel of $18 \mu \mathrm{m}$ length and a width of $1 \mu \mathrm{m}$ in one case and $4 \mu \mathrm{m}$ in the other. On both sides of the channel two point contacts are defined, with 3- $\mu \mathrm{m}$ separation. A schematic layout of the gates and contacts is given in Fig. 1(a). Resistance measurements on these samples are made using phasesensitive techniques. The samples are kept at $1.8 \mathrm{~K}$ in a cryostat equipped with a superconducting magnet.

The relevant quantity regarding the degree of collimation in our devices is the increase in $T_{i \rightarrow c}$, the transmission probability for electrons to travel directly from one point contact, the injector $i$, to the opposite point contact, the collector $c$. From the semiclassical analysis given in
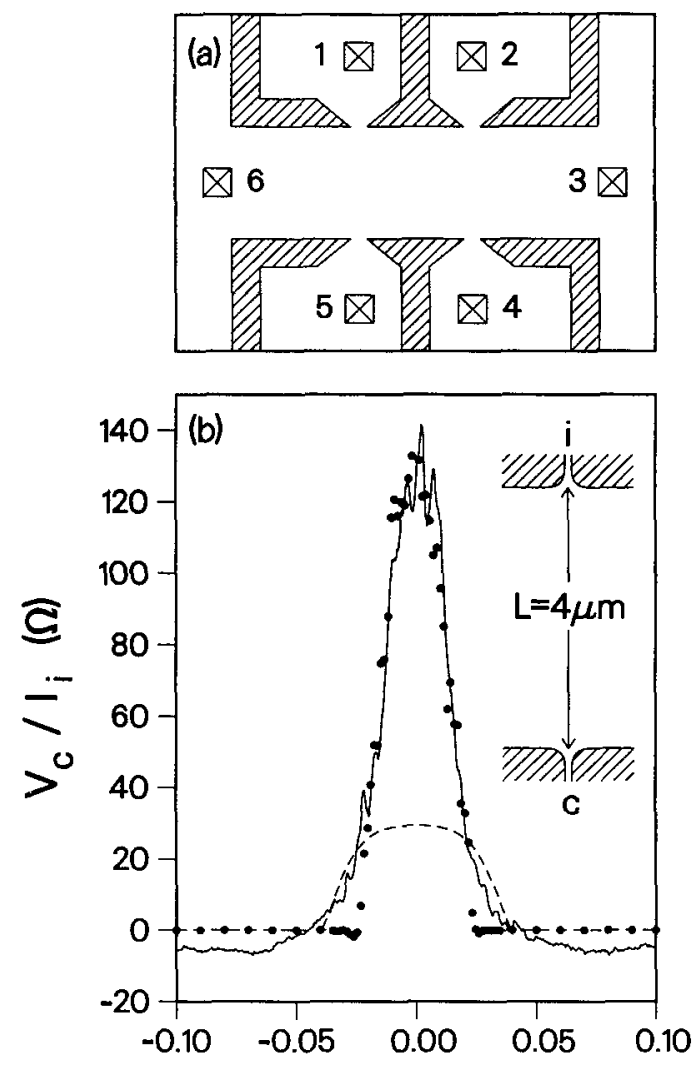

Magnetic Field (T)

FIG. 1. (a) The sample layout. The dashed areas indicate the gates; the squares are Ohmic contacts to the 2D EG. (b) Plots of $V_{c} / I_{i}=R_{16,53}$ vs magnetic field for the device with a channel width of $L=4.0 \mu \mathrm{m}$. The drawn line is the experimental curve. The filled circles are the results of the simulations described in the text, using the hard-wall potential shown in the inset (the simulated device contains two sets of opposite point contacts, a distance of $3.0 \mu \mathrm{m}$ apart); the dashed line results from simulations with rectangular corners in the potential contour (no collimation). 
Ref. 2 we obtain that, in the presence of collimation,

$$
T_{t \rightarrow c}=f^{2} \frac{\pi}{2 k_{F} L} N^{2} \text {. }
$$

Here, $L$ is the distance between two opposite identical point contacts, $k_{F}$ is the Fermi wave vector in the 2D EG between the point contacts, and $N$ is the number of quantum channels (or occupied subbands) in each point contact. The factor $f \geq 1$ describes the collimation in the point contacts in the approximation of adiabatic transport in the point-contact region; two phenomena contribute to its magnitude, i.e., the flaring of the potential boundary of the point contact from a width $W_{\min }$ to $W_{\max }$, and the presence of a barrier of height $E_{0}$ in the point contact. As shown in Ref. 2,

$$
f=\frac{W_{\max }}{W_{\min }} \frac{k_{F}}{k_{\mathrm{pc}}},
$$

where $k_{\mathrm{pc}} \equiv\left[2 m\left(E_{F}-E_{0}\right) / \hbar^{2}\right]^{1 / 2}$ is the Fermi wave vector in the point contact. Note that $N=k_{\mathrm{pc}} W_{\mathrm{min}} / \pi$. In addition to the assumption of adiabatic transport, Eq. (1) also assumes that $T_{l \rightarrow c} \ll N$, which requires $f W_{\max } / L$ $\ll 1$. Both of these two assumptions will be relaxed below, when we consider the classical simulation method.

We perform four-terminal magnetoresistance measurements in a generalized longitudinal geometry ${ }^{6}$ that allows a direct determination of the collimation factor $f$ of a point contact. The gate voltages are adjusted to yield equal resistance values for the injector and collector point contact, which should also lead to approximately equal collimation factors [cf. Eq. (4)]. The current $I_{l}$ is injected through point contact 1 and flows to drain contact 6; the collector voltage $V_{c}$ on contact 5 is measured relative to the voltage at contact 3 . The measured resistance is $V_{c} / I_{l}$, which is $R_{16,53}$ in the generally accepted notation. ${ }^{6,7}$ Using the Büttiker formula ${ }^{7}$ and the approximations leading to Eq. (1), one finds that at zero magnetic field $B$,

$$
\frac{V_{c}}{I_{l}}(B=0)=\frac{h}{2 e^{2}}\left(f^{2}-\frac{1}{2}\right) \frac{\pi}{2 k_{F} L} .
$$

Since $k_{F}$ and $L$ are known, the collector voltage is a direct measure of the degree of collimation. For nonzero magnetic fields, the adiabatic theory predicts a slowly decreasing signal as the injected electron beam is deflected; the signal abruptly falls to zero when the beam is fully swept past the collector contact. This occurs when $2 l_{\text {cycl }} / L=f$, where $l_{\text {cycl }} \equiv \hbar k_{F} / e B$ is the cyclotron radius in the channel.

In Fig. 1(b) we show an experimental trace (the drawn curve) of $V_{c} / I_{l}$ vs $B$ for a device with an injector-collector separation $L=4 \mu \mathrm{m}$. The point-contact resistance is 2.8 $k \Omega$. From Shubnikov-de Haas data we estimate $k_{F}=1.1 \times 10^{8} \mathrm{~m}^{-1}$. From the zero-field amplitude of the collector signal we derive, using Eq. (3), a collimation factor of $f=1.85$, which is larger than 1 , providing direct evidence of the occurrence of collimation.

Assuming adiabatic transport in the point contact, ${ }^{2}$ the full opening angle $\Delta a$ of the emerging electron beam can be related to $f$ by $\Delta \alpha=2 \arcsin (1 / f)$. The angular distribution $P(\alpha)$ of the injected electrons (with $\alpha$ the angle with the axis connecting both point contacts) is then $P(\alpha)=\frac{1}{2} f \cos \alpha$ for $|\alpha|<\Delta \alpha / 2$, and $P(\alpha)=0$ elsewise.
In Fig. 2, the dotted line gives this angular distribution, with $\Delta \alpha=65^{\circ}$ for the experimental value of $f=1.85$.

The above formulas assume adiabatic transport in the point contact and a small transmission probability $T_{t \rightarrow c}$. To relax these assumptions, we have carried out a simulation of classical electron trajectories in an appropriate potential landscape, using the methods of Ref. 8. We have defined the four point contacts using a hard-wall potential with contours shown in the inset of Fig. 1(b). The minimal width of the point contacts is fixed at $100 \mathrm{~nm}$ (roughly consistent with the measured point-contact resistance). No potential barriers are included, so that all collimation is due to the horn effect, i.e., the flaring at the exit and entrance of the point contact. We first carried out a simulation for the case of no collimation, using point contacts with rectangular corners. The result is the dashed curve in Fig. 1(b), which is clearly in gross disagreement with the experimental data. Good agreement could be obtained with a moderate degree of flaring (the contours in the inset are drawn to scale), as shown by the filled circles.

As is evident from Fig. 1, the overall shape (magnitude and width) of the experimental trace is well reproduced by the simulation. The experimental peak shows a tail and a small offset at higher fields that are not found in the simulation, most likely the results of a diffuse background resistance. More interestingly, the experimental trace shows fine structure at the top which is not found classically, and which we attribute to quantum interference effects.

In Fig. 2 we show the simulated angular distribution of the injected beam (solid curve), which is somewhat broader than the result of the adiabatic approximation

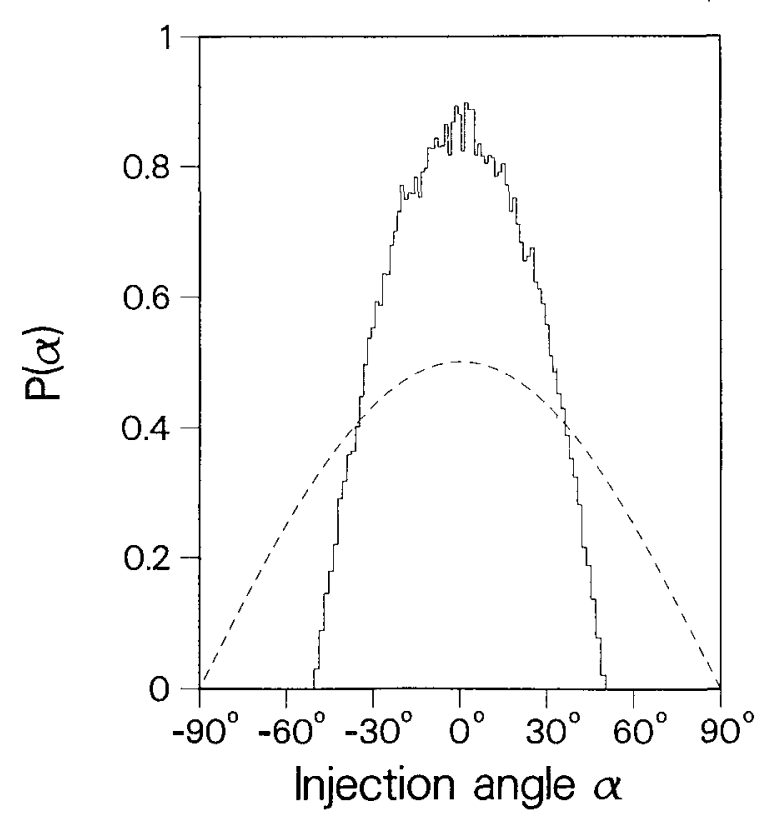

FIG. 2. Distribution of injection angles (at zero field) for injected electrons obtained from the simulation in Fig. 1(b) (solid line). The dotted line is the result of the adiabatic approximation using $f=1.85$; the dashed line is the angular distribution in absence of collimation. 
discussed above (dotted curve). Both curves are much narrower than the distribution $P(\alpha)=\frac{1}{2} \cos \alpha$ of an uncollimated beam (the dashed curve). From the full width at half maximum of the simulated distribution we find a characteristic opening angle of $70^{\circ}$, quite close to the value obtained from the adiabatic approximation.

The degree of collimation in our devices can be varied by adjusting the gate voltage. In Fig. 3, we have plotted the collimation factor $f$ [obtained using Eq. (3)] versus the point-contact resistance $R_{\mathrm{pc}}$ for one $L=1 \mu \mathrm{m}$ and two $L=4 \mu \mathrm{m}$ devices. (The value of $k_{F}$ is approximately the same in all three devices.) One expects ${ }^{2}$ a strong increase in collimation at more negative gate voltages, because both collimation mechanisms are enhanced on narrowing the point-contact width, i.e., the potential barrier in the point contact will increase and the horn shape of the potential contour will be more pronounced. More precisely, combining Eq. (2) and the formula for the point-contact resistance $\left[R_{\mathrm{pc}}=h / 2 e^{2} N=\left(h / 2 e^{2}\right)\left(\pi / k_{\mathrm{pc}} W_{\min }\right)\right] \quad$ one finds

$$
f=R_{\mathrm{pc}} \frac{2 e^{2}}{h} \frac{k_{F} W_{\max }}{\pi} .
$$

Since $W_{\max }$ is expected to be relatively constant and device independent, one would expect an approximately linear dependence of $f$ on $R_{\mathrm{pc}}$. This is indeed observed; see Fig. 3. The dashed line in this figure is from Eq. (4), with $W_{\max }=270 \mathrm{~nm}$ and the measured value $k_{F}=1.1$ $\times 10^{8} \mathrm{~m}^{-1}$. This value for $W_{\max }$ is about equal to the lithographic opening in the split gate defining the point contact, which is not unreasonable. We find it remarkable that the simple formula (4), with one set of parameters, can describe the collimation effects in three different devices to within about $30 \%$. At high values of $R_{\mathrm{pc}}$, a semiclassical treatment of collimation in point contacts is no longer expected to be valid, since the width approaches the Fermi wavelength of the electrons. We should add here that the occurrence of collimation at the relatively wide $(W \gtrsim 100 \mathrm{~nm})$ point contacts found here implies that the main effect is the flaring of the potential boundaries - one does not expect strong barrier collimation for the low gate voltages involved (i.e., $k_{F} / k_{\mathrm{pc}} \approx 1$ ).

In addition to the experiment with directly opposite injector and collector point contacts (i.e., a measurement of $R_{16,53}$, we have also measured $R_{16,43}$, with diagonally opposite point contacts. The numerical simulations predict that this signal shows a peak around zero magnetic field, of comparable height and width to the peak in $R_{16,53}$. The peak is symmetric in $B$ because of reciprocity ${ }^{7}\left[R_{16,43}(B)\right.$ $\left.=R_{43,16}(-B)\right]$, and the symmetric device layout. This peak is due to electrons that leave the injector almost perpendicularly to the channel wall, and arrive at the collector after a large number of specular reflections. Experimentally, such a peak is indeed observed, but its zero-field amplitude [ca. $20 \Omega$ for the parameters of Fig. 1(b)] is almost an order of magnitude smaller than predicted (120 $\Omega)$. The $L=1 \mu$ m-device yields similarly small peak

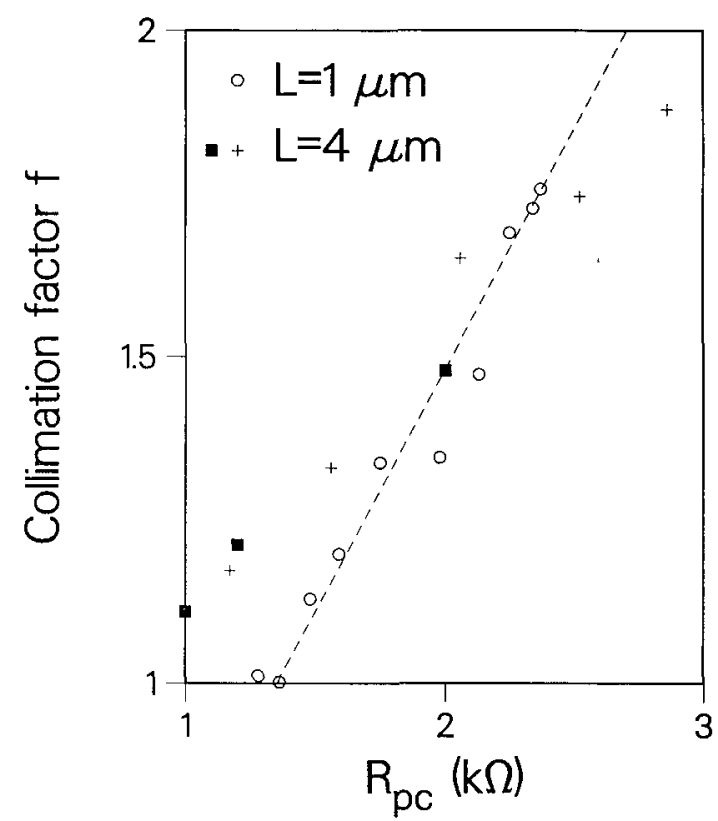

FIG. 3. The dependence of the collimation factor $f$ on the point-contact resistance $R_{\mathrm{pc}}$ (injector and collector point contact are adjusted to equal resistances), for three different devices. The collimation factor is calculated, using Eq. (3), from the zero-field value of $V_{c} / I_{1}$, relative to the residual resistance at high magnetic fields. The dashed line is the result of the adiabatic approximation [Eq. (4)], using $W_{\max }=270 \mathrm{~nm}$ and the measured value $k_{F}=1.1 \times 10^{8} \mathrm{~m}^{-1}$.

heights for $R_{16,43}(B=0)$. A possible explanation for the discrepancy between predicted and observed amplitude of the peak in $R_{16,43}$ is the occurrence of small deviations from straightness in the channel walls, which destroy collimation after many reflections. ${ }^{9}$ Another possibility is impurity scattering, which would presumably affect the size of $R_{16,43}$ more than that of $R_{16,53}$ because of the longer path length involved.

In conclusion, we have demonstrated that collimation occurs for electrons injected through a narrow point contact and can be maintained over distances of at least 4 $\mu \mathrm{m}$. The degree of collimation can be varied by adjusting the gate voltage, and scales linearly with the point-contact resistance. The effect can be well understood by a semiclassical description, either analytically (using the adiabatic approximation ${ }^{2}$ ) or by a numerical simulation. The occurrence of collimation and, concomitantly, of maximum injection and acceptance angles, is an effect that should be carefully taken into account when interpreting transport phenomena in narrow channels. ${ }^{3,8}$

We wish to thank M. E. I. Broekaart and S. Phelps for their contributions towards the sample fabrication and $\mathrm{H}$. van Houten and M. F. H. Schuurmans for valuable discussions. 
Ref. 2 we obtain that, in the presence of collimation,

$$
T_{l \rightarrow c}=f^{2} \frac{\pi}{2 k_{F} L} N^{2} .
$$

Here, $L$ is the distance between two opposite identical point contacts, $k_{F}$ is the Fermi wave vector in the 2D EG between the point contacts, and $N$ is the number of quantum channels (or occupied subbands) in each point contact. The factor $f \geq 1$ describes the collimation in the point contacts in the approximation of adiabatic transport in the point-contact region; two phenomena contribute to its magnitude, i.e., the flaring of the potential boundary of the point contact from a width $W_{\min }$ to $W_{\max }$, and the presence of a barrier of height $E_{0}$ in the point contact. As shown in Ref. 2,

$$
f=\frac{W_{\max }}{W_{\min }} \frac{k_{F}}{k_{\mathrm{pc}}},
$$

where $k_{\mathrm{pc}} \equiv\left[2 m\left(E_{F}-E_{0}\right) / \hbar^{2}\right]^{1 / 2}$ is the Fermi wave vector in the point contact. Note that $N=k_{\mathrm{pc}} W_{\min } / \pi$. In addition to the assumption of adiabatic transport, Eq. (1) also assumes that $T_{t \rightarrow c} \ll N$, which requires $f W_{\max } / L$ $\ll 1$. Both of these two assumptions will be relaxed below, when we consider the classical simulation method.

We perform four-terminal magnetoresistance measurements in a generalized longitudinal geometry ${ }^{6}$ that allows a direct determination of the collimation factor $f$ of a point contact. The gate voltages are adjusted to yield equal resistance values for the injector and collector point contact, which should also lead to approximately equal collimation factors [cf. Eq. (4)]. The current $I_{l}$ is injected through point contact 1 and flows to drain contact 6 ; the collector voltage $V_{c}$ on contact 5 is measured relative to the voltage at contact 3 . The measured resistance is $V_{c} / I_{l}$, which is $R_{16,53}$ in the generally accepted notation. ${ }^{6,7}$ Using the Büttiker formula ${ }^{7}$ and the approximations leading to Eq. (1), one finds that at zero magnetic field $B$,

$$
\frac{V_{c}}{I_{l}}(B=0)=\frac{h}{2 e^{2}}\left(f^{2}-\frac{1}{2}\right) \frac{\pi}{2 k_{F} L} .
$$

Since $k_{F}$ and $L$ are known, the collector voltage is a direct measure of the degree of collimation. For nonzero magnetic fields, the adiabatic theory predicts a slowly decreasing signal as the injected electron beam is deflected; the signal abruptly falls to zero when the beam is fully swept past the collector contact. This occurs when $2 l_{\text {cycl }} / L=f$, where $l_{\text {cycl }} \equiv \hbar k_{F} / e B$ is the cyclotron radius in the channel.

In Fig. 1(b) we show an experimental trace (the drawn curve) of $V_{c} / I_{i}$ vs $B$ for a device with an injector-collector separation $L=4 \mu \mathrm{m}$. The point-contact resistance is 2.8 $\mathrm{k} \Omega$. From Shubnikov-de Haas data we estimate $k_{F}=1.1 \times 10^{8} \mathrm{~m}^{-1}$. From the zero-field amplitude of the collector signal we derive, using Eq. (3), a collimation factor of $f=1.85$, which is larger than 1 , providing direct evidence of the occurrence of collimation.

Assuming adiabatic transport in the point contact, ${ }^{2}$ the full opening angle $\Delta \alpha$ of the emerging electron beam can be related to $f$ by $\Delta \alpha=2 \arcsin (1 / f)$. The angular distribution $P(\alpha)$ of the injected electrons (with $\alpha$ the angle with the axis connecting both point contacts) is then $P(\alpha)=\frac{1}{2} f \cos \alpha$ for $|\alpha|<\Delta \alpha / 2$, and $P(\alpha)=0$ elsewise.
In Fig. 2, the dotted line gives this angular distribution, with $\Delta \alpha=65^{\circ}$ for the experimental value of $f=1.85$.

The above formulas assume adiabatic transport in the point contact and a small transmission probability $T_{i \rightarrow c}$. To relax these assumptions, we have carried out a simulation of classical electron trajectories in an appropriate potential landscape, using the methods of Ref. 8. We have defined the four point contacts using a hard-wall potential with contours shown in the inset of Fig. 1(b). The minimal width of the point contacts is fixed at $100 \mathrm{~nm}$ (roughly consistent with the measured point-contact resistance). No potential barriers are included, so that all collimation is due to the horn effect, i.e., the flaring at the exit and entrance of the point contact. We first carried out a simulation for the case of no collimation, using point contacts with rectangular corners. The result is the dashed curve in Fig. 1(b), which is clearly in gross disagreement with the experimental data. Good agreement could be obtained with a moderate degree of flaring (the contours in the inset are drawn to scale), as shown by the filled circles.

As is evident from Fig. 1, the overall shape (magnitude and width) of the experimental trace is well reproduced by the simulation. The experimental peak shows a tail and a small offset at higher fields that are not found in the simulation, most likely the results of a diffuse background resistance. More interestingly, the experimental trace shows fine structure at the top which is not found classically, and which we attribute to quantum interference effects.

In Fig. 2 we show the simulated angular distribution of the injected beam (solid curve), which is somewhat broader than the result of the adiabatic approximation

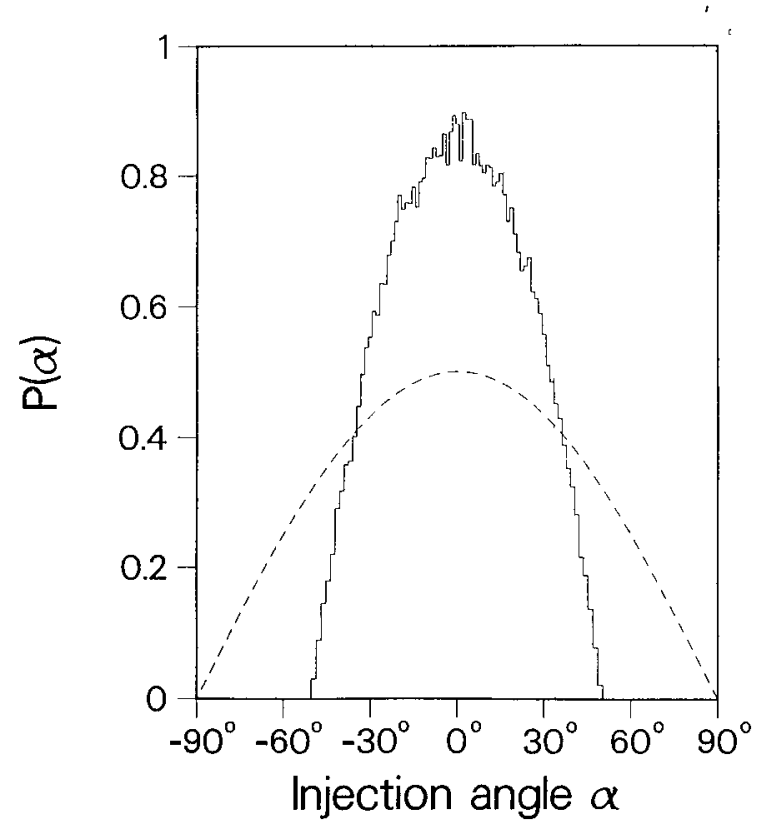

FIG. 2. Distribution of injection angles (at zero field) for injected electrons obtained from the simulation in Fig. 1(b) (solid line). The dotted line is the result of the adiabatic approximation using $f=1.85$; the dashed line is the angular distribution in absence of collimation. 
discussed above (dotted curve). Both curves are much narrower than the distribution $P(\alpha)=\frac{1}{2} \cos \alpha$ of an uncollimated beam (the dashed curve). From the full width at half maximum of the simulated distribution we find a characteristic opening angle of $70^{\circ}$, quite close to the value obtained from the adiabatic approximation.

The degree of collimation in our devices can be varied by adjusting the gate voltage. In Fig. 3, we have plotted the collimation factor $f$ [obtained using Eq. (3)] versus the point-contact resistance $R_{\mathrm{pc}}$ for one $L=1 \mu \mathrm{m}$ and two $L=4 \mu \mathrm{m}$ devices. (The value of $k_{F}$ is approximately the same in all three devices.) One expects ${ }^{2}$ a strong increase in collimation at more negative gate voltages, because both collimation mechanisms are enhanced on narrowing the point-contact width, i.e., the potential barrier in the point contact will increase and the horn shape of the potential contour will be more pronounced. More precisely, combining Eq. (2) and the formula for the point-contact resistance $\left[R_{\mathrm{pc}}=h / 2 e^{2} N=\left(h / 2 e^{2}\right)\left(\pi / k_{\mathrm{pc}} W_{\min }\right)\right] \quad$ one finds

$$
f=R_{\mathrm{pc}} \frac{2 e^{2}}{h} \frac{k_{F} W_{\max }}{\pi} .
$$

Since $W_{\max }$ is expected to be relatively constant and device independent, one would expect an approximately linear dependence of $f$ on $R_{\mathrm{pc}}$. This is indeed observed; see Fig. 3. The dashed line in this figure is from Eq. (4), with $W_{\max }=270 \mathrm{~nm}$ and the measured value $k_{F}=1.1$ $\times 10^{8} \mathrm{~m}^{-1}$. This value for $W_{\max }$ is about equal to the lithographic opening in the split gate defining the point contact, which is not unreasonable. We find it remarkable that the simple formula (4), with one set of parameters, can describe the collimation effects in three different devices to within about $30 \%$. At high values of $R_{\mathrm{pc}}$, a semiclassical treatment of collimation in point contacts is no longer expected to be valid, since the width approaches the Fermi wavelength of the electrons. We should add here that the occurrence of collimation at the relatively wide $(W \gtrsim 100 \mathrm{~nm}$ ) point contacts found here implies that the main effect is the flaring of the potential boundaries - one does not expect strong barrier collimation for the low gate voltages involved (i.e., $k_{F} / k_{\mathrm{pc}} \simeq 1$ ).

In addition to the experiment with directly opposite injector and collector point contacts (i.e., a measurement of $R_{16,53}$ ), we have also measured $R_{16,43}$, with diagonally opposite point contacts. The numerical simulations predict that this signal shows a peak around zero magnetic field, of comparable height and width to the peak in $R_{16,53}$. The peak is symmetric in $B$ because of reciprocity ${ }^{7}\left[R_{16,43}(B)\right.$ $=R_{43,16}(-B)$ ], and the symmetric device layout. This peak is due to electrons that leave the injector almost perpendicularly to the channel wall, and arrive at the collector after a large number of specular reflections. Experimentally, such a peak is indeed observed, but its zero-field amplitude [ca. $20 \Omega$ for the parameters of Fig. 1(b)] is almost an order of magnitude smaller than predicted ( 120 $\Omega$ ). The $L=1 \mu \mathrm{m}$-device yields similarly small peak

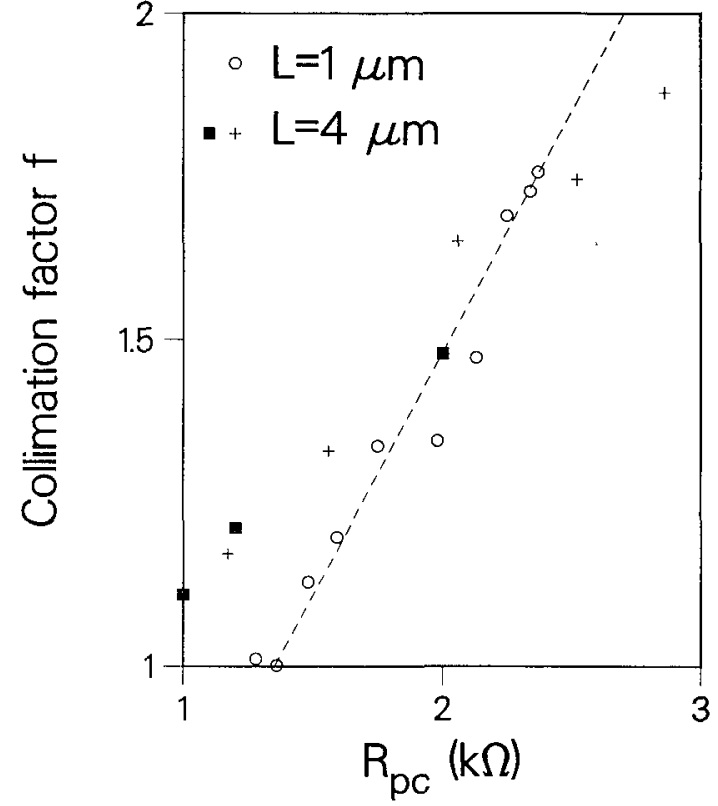

FIG. 3. The dependence of the collimation factor $f$ on the point-contact resistance $R_{\mathrm{pc}}$ (injector and collector point contact are adjusted to equal resistances), for three different devices. The collimation factor is calculated, using Eq. (3), from the zero-field value of $V_{c} / I_{i}$, relative to the residual resistance at high magnetic fields. The dashed line is the result of the adiabatic approximation [Eq. (4)], using $W_{\max }=270 \mathrm{~nm}$ and the measured value $k_{F}=1.1 \times 10^{8} \mathrm{~m}^{-1}$.

heights for $R_{16,43}(B=0)$. A possible explanation for the discrepancy between predicted and observed amplitude of the peak in $R_{16,43}$ is the occurrence of small deviations from straightness in the channel walls, which destroy collimation after many reflections. ${ }^{9}$ Another possibility is impurity scattering, which would presumably affect the size of $R_{16,43}$ more than that of $R_{16,53}$ because of the longer path length involved.

In conclusion, we have demonstrated that collimation occurs for electrons injected through a narrow point contact and can be maintained over distances of at least 4 $\mu \mathrm{m}$. The degree of collimation can be varied by adjusting the gate voltage, and scales linearly with the point-contact resistance. The effect can be well understood by a semiclassical description, either analytically (using the adiabatic approximation ${ }^{2}$ ) or by a numerical simulation. The occurrence of collimation and, concomitantly, of maximum injection and acceptance angles, is an effect that should be carefully taken into account when interpreting transport phenomena in narrow channels. ${ }^{3,8}$

We wish to thank M. E. I. Broekaart and S. Phelps for their contributions towards the sample fabrication and $\mathrm{H}$. van Houten and M. F. H. Schuurmans for valuable discussions. 
*Permanent address: Eindhoven University of Technology, 5600 MB Eindhoven, The Netherlands.

${ }^{1}$ D. A. Wharam, M. Pepper, H. Ahmed, J. E. F. Frost, D. G. Hasko, D. C. Peacock, D. A. Ritchie, and G. A. C. Jones, J. Phys. C 21, L887 (1988).

${ }^{2}$ C. W. J. Beenakker and H. van Houten, Phys. Rev. B 39, 10445 (1989); see also H. van Houten and C. W. J. Beenakker, in Nanostructure Physics and Fabrication, edited by $M$. A. Reed and W. P. Kirk (Academic, New York, 1989), p. 347.

${ }^{3}$ H. U. Baranger and A. D. Stone, Phys. Rev. Lett. 63, 414 (1989).

${ }^{4}$ M. L. Roukes, A. Scherer, S. J. Allen, Jr., H. G. Craighead, R. M. Ruthen, E. D. Beebe, and J. P. Harbison, Phys. Rev. Lett. 59, 3011 (1987).

${ }^{5}$ A. M. Chang, T. Y. Chang, and H. U. Baranger, Phys. Rev. Lett. 63, 996 (1989).

${ }^{6}$ G. Timp, H. U. Baranger, P. de Vegvar, J. E. Cunningham, R. E. Howard, R. Behringer, and P. M. Mankiewich, Phys. Rev. Lett. 60, 2081 (1988); Y. Takagaki, K. Gamo, S. Namba, S. Ishida, S. Takaoka, K. Murase, K. Ishibasi, and Y. Aoyagi, Solid State Commun. 68, 1051 (1988).

${ }^{7}$ M. Büttiker, IBM J. Res. Dev. 32, 317 (1988).

${ }^{8}$ C. W. J. Beenakker and H. van Houten, Phys. Rev. Lett. 63, 1857 (1989).

${ }^{9}$ The anomalously small peak in $R_{16,43}$ may well be related to the anomalously small nonlocal bend resistance observed by Timp et al. (Ref. 6) in a narrow channel, which was attributed to small-angle impurity scattering. Scattering from channel wall irregularities would also destroy the nonequilibrium velocity distribution responsible for the bend resistance effect, without appreciably reducing the mobility, and may therefore be an additional reason for the anomaly. 